\title{
Planetary Nebula Luminosity Functions and Prospects for the Future
}

\author{
ROBIN CIARDULLO \\ The Pennsylvania State University
}

\begin{abstract}
Until now, [O III] $\lambda 5007$ surveys of extragalactic planetary nebulae (PN) have been aimed at determining distances and identifying test particles for future kinematical studies. However, PN observations are useful in another area of astrophysics - the investigation of stellar populations. Because the production rate of [O III] bright $P N$ in a galaxy can vary by an order of magnitude, this number is potentially a powerful discriminant between age and metallicity in an evolved stellar population. A strong inverse correlation exists between galaxy absolute magnitude and bolometric luminosity-specific PN density $\left(\alpha_{2.5}\right)$, with the small metal-poor ellipticals having values of $\alpha_{2.5}$ in exact accordance with the predictions of stellar evolution theory. This correlation, along with the direct dependence of $\alpha_{2.5}$ on galactocentric radius, and the scatter in $\alpha_{2.5}$ seen in "young" galaxies, argues strongly that elliptical galaxies are old systems which follow a metallicity sequence. It also suggests that the combination of absorption line spectroscopy, UV photometry, and $P N$ measurements can be a powerful probe of elliptical galaxy stellar populations.
\end{abstract}

Key words: galaxies: elliptical and lenticular - galaxies: stellar content

[O III] $\lambda 5007$ surveys of extragalactic planetary nebulae are enormously useful in astrophysics. First, the cutoff in a galaxy's planetary nebula luminosity function (PNLF) is an excellent standard candle. Second, PN are wonderful test particles for stellar kinematics: by performing spectroscopy on the PN identified in imaging surveys, it is possible to look for dark matter far out in a galaxy's halo. Finally, the luminosity-specific PN density provides an important constraint on a stellar population's metallicity and age. Although much attention has been devoted to using PN as distance indicators and dynamical probes, the ability of PN to probe elliptical galaxy evolution has not yet been exploited. Here we examine this possibility.

Figure 1 compares the combined PNLF of 22 galaxies with the law proposed by Ciardullo et al. (1989), $N(M) \propto e^{0.307 M}\left\{1-e^{3\left(M^{*}-M\right)}\right\}$. Clearly, the PNLF cutoff is approximated well (but not perfectly) by the empirical function. Since the PNLF distances derived to date have all assumed this simple law, errors in the adopted form of the PNLF propagate directly into errors in distance. Fortunately, as the figure demonstrates, the true PNLF agrees well with the model.

Also visible in Figure 1 is the small, but prominent population of overluminous PN. Less than $1 \%$ of all extragalactic PN fall into this category, yet these objects present a serious challenge for PNLF modeling. Because overluminous PN are present in old, gas-poor stellar populations, they are probably not compact H II regions, Wolf-Rayet nebulae, or supernova remnants. (None of the 6 recorded supernovae in our surveyed galaxies have been recovered in $\lambda 5007$.) Similarly, although some overluminous PN can be modeled as chance superpositions of bright objects (Jacoby, Ciardullo, \& Ford 1990) or PN with massive central stars (Méndez et al. 1993), such explanations do not work for the brightest sources. One possible mechanism for producing overluminous PN is interacting binary stars. Di Stefano, Paerels, \& Rappaport (1995) point out that the supersoft x-ray sources found by ROSAT have the proper temperatures and luminosities to produce overluminous PN. Moreover, despite the small number of supersoft sources detected, these objects must be fairly common (Di Stefano \& Rappaport 1994), and one bright PN, N67 


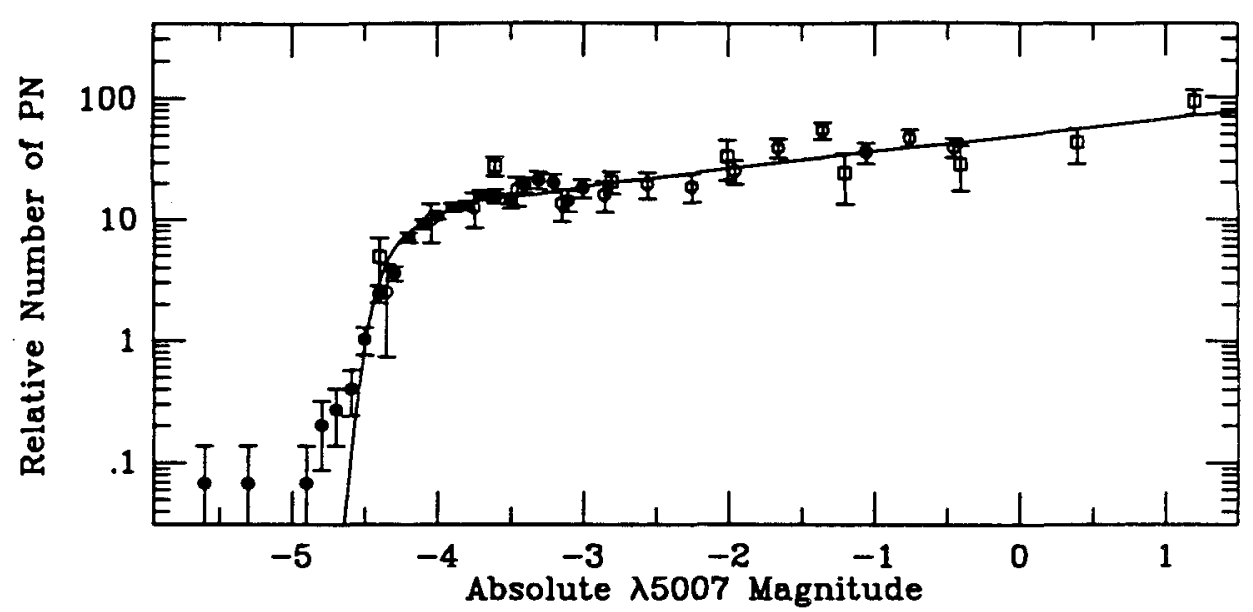

Fig. 1. The solid points show the [O III] $\lambda 5007$ PNLF formed from statistically complete samples of PN in 20 galaxies. The open circles give the PNLF of M31's bulge; the open squares show the PNLF of the LMC. The solid line is the empirical law convolved with a function representing photometric errors. Optical doubling is not included in the model.

in the SMC, is coincident with a supersoft source (Brown et al. 1994). Still, it is not clear how these objects can be surrounded by a dense, compact nebula.

Perhaps the most underappreciated feature of Figure 1 is the agreement between the faint end of the PNLF and the model curve. The PN of the LMC and M31's bulge have very different parent populations, yet both PNLFs agree perfectly with that predicted from an ensemble of uniform expanding nebulae with non-evolving central stars. This suggests that large numbers of PN are not entering the PNLF at faint magnitudes, and implies that the total number of PN in a galaxy can be derived simply by integrating the empirical curve over its entire $\sim 8$ mag range.

Renzini \& Buzzoni (1986) have shown that in non-star-forming stellar populations, the bolometric-luminosity specific stellar evolutionary flux should be about $2 \times 10^{-11}$ stars-yr ${ }^{-1}-L_{\odot}^{-1}$, independent of population age or initial mass function. If the lifetime of the planetary nebula stage is $\sim 25,000 \mathrm{yr}$, then galaxies should have $\sim 50 \times 10^{-8} \mathrm{PN}-L_{\odot}^{-1}$. The empirical function then implies that one-tenth of these PN should be within $2.5 \mathrm{mag}$ of $M^{*}$.

Figure 2 shows the bolometric-luminosity specific PN density $\left(\alpha_{2.5}\right)$ of 23 earlytype galaxies. The figure demonstrates that there is a hard upper limit to $\alpha_{2.5}$ which is in perfect agreement with theory, but that the PN population in some galaxies is down by almost an order of magnitude. For bright galaxies, $\alpha_{2.5}$ correlates strongly with galaxy absolute magnitude, with large galaxies being PN deficient. PN density is also inversely correlated with galaxy UV flux, which suggests that the missing PN are AGB manque oljects (Greggio \& Renzini 1990). Significantly, these variations cannot be due solely to population age: in six galaxies with young or intermediate age stars, $\alpha_{2.5}$ varies by a factor of five. Instead, the evidence suggests that $\alpha_{2.5}$ depends on metallicity. This interpretation is supported by 1) an inverse correlation 


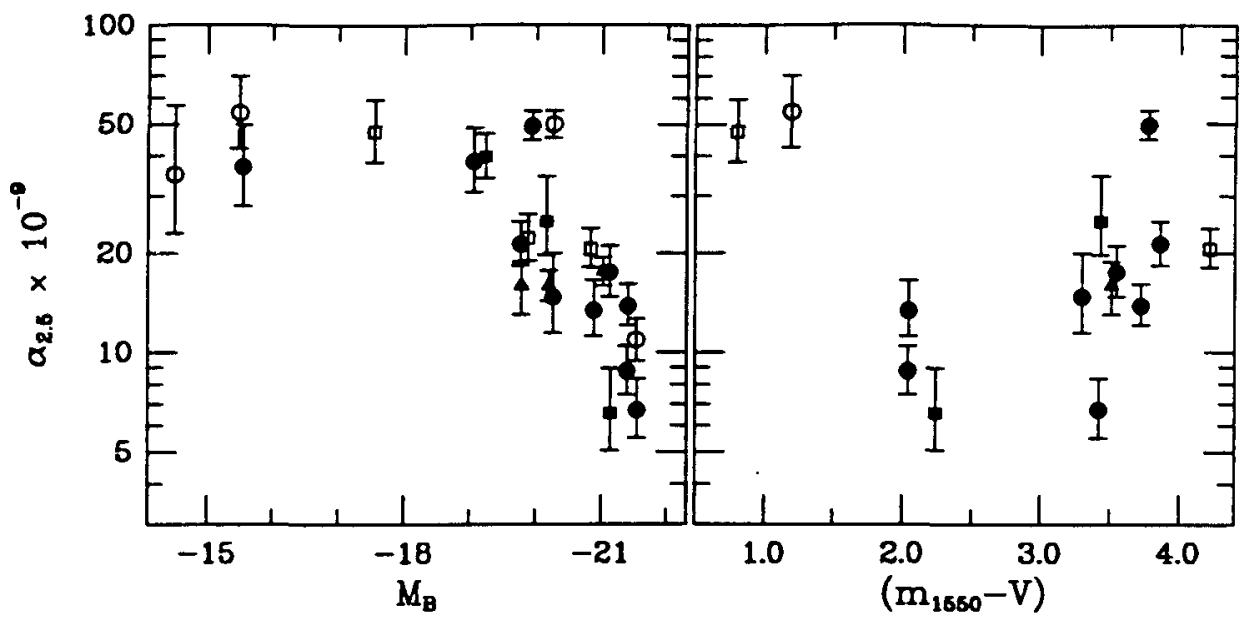

Fig. 2. Correlations between $\alpha_{2.5}$, galaxy absolute $B$ magnitude, and UV color. Circles are elliptical galaxies, squares are lenticulars, and triangles are spiral bulges. Open symbols show galaxies with presumed "young" populations, as evidenced by either on-going star formation or blue colors and morphological peculiarities. The error bars represent formal $1 \sigma$ uncertainties and are lower limits to the true errors.

between $\alpha_{2.5}$ and $\mathrm{Mg}_{2}$ index, 2) a direct correlation between $\alpha_{2.5}$ and galactocentric distance in a synthetic galaxy formed from eight normal ellipticals, and 3) a direct correlation between $\alpha_{2.5}$ and galactocentric distance in NGC 5128 (Hui et al. 1993).

If the correlations of Figure 2 were entirely due to metallicity, then $\alpha_{2.5}$ would reflect the fraction of super-metal rich stars in a galaxy, and would be an invaluable constraint on chemical evolution. However, the very small values of $\alpha_{2.5}$ found in some galaxies suggests that age may also play a role in determining the PN population, perhaps by shortening the nebula lifetimes of PN with low-mass progenitors. (When ejected, the low-mass envelopes of these stars should disperse rather quickly.) Nevertheless, the large variation of $\alpha_{2.5}$ presents us with an important tool for probing stellar populations. By combining PNLF measurements with UV photometry and absorption line analyses, it should be possible to break the age-metallicity degeneracy for elliptical galaxies and produce realistic models for galactic evolution.

\section{References}

Brown, T., Córdova, F., Ciardullo, R., Thompson, R., and Bond, H. 1994, A p. J., $422,118$.

Ciardullo, R., Jacoby, G.H., Ford, H.C., \& Neill, J.D. 1989, Ap. J., 339, 53.

Di Stefano, R., Paerels, F., \& Rappaport, S. 1995, in preparation.

Di Stefano, R., \& Rappaport, S. 1994, A p. J., in press.

Greggio, L., \& Renzini, A. 1990, Ap. J., 364, 35.

Hui, X., Ford, H.C., Cinrtullo, R., \& Jacoby, G.H. 1993, Ap. J., 414, 463.

Jacoby, G.H., Ciardullo, R., \& Ford, H.C. 1990, A p. J., 356, 332.

Méndez, R.H., Kultrizki, R.P., Ciardullo, R., \& Jacoby, G.H. 1993, A str. A p., 414, 454.

Renzini, A., \& Bumoni, A. 1986, in Spectral Evolution of Galaxies, ed. C. Chiosi \& A. Renzini,

(Dordrecht: Reiclel), p. 195. 